\title{
Performance Characterization of Modal Identification Algorithms, the case of Automated Modal Analysis of Palazzo Lombardia
}

\author{
Marcello Vanali ${ }^{1}$, Marta Berardengo ${ }^{1}$, Stefano Manzoni ${ }^{2}$, Giorgio Busca $^{2}$, Elena Mola ${ }^{3}$ \\ 1 Dipartimento di Ingegneria Industriale, Università degli studi di Parma \\ 2 Dipartimento di Meccanica, Politecnico di Milano
}

3 ECSD Srl, Milano

\begin{abstract}
In this paper the study of the analysis of the data coming from the permanent monitoring system of Palazzo Regione Lombardia, one of the tallest buildings in Milano is presented. The system works acquiring both the vibrations of the building and its static behaviour. The vibration data are used to carry out continuous modal analysis to the aim of detecting possible changes of the structure. Two algorithms for modal parameter analysis are presented and compared through Monte Carlo simulations. The results of this comparison allowed to choose the best data analysis procedure to be used on the real data measured under operating conditions. The first results coming from the modal parameter extraction are presented.
\end{abstract}

KEYWORDS: OMA, Health Monitoring, Vibration, Uncertainty estimation, Continuous monitoring

\section{INTRODUCTION}

In recent times the monitoring of civil structures has become more and more interesting and affordable thanks to the continuous technological development. Measurement devices and transducers have become cheaper and cheaper, together with the improvement of their metrological characteristics, while data acquisition and storage system are available as off the shelf solutions. This allows to instrument huge structures with many sensors with different aims: (i) the prompt detection of changes of the structure behaviour, (ii) the monitoring of comfort of people living/working in the monitored building, (iii) the check of the structure behaviour after exceptional events such as earthquakes or severe thuderstorms.

Continuous monitoring of civil structures still requires to face a number of challenges: e.g. efficient management and visualization of the data requires implementing a tailored data pre-processing, the check of sensor stability over long periods of time. Nowadays the redundancy of transducers prevents loss of data on one side, but also requires to find synthetic indexes and features able to provide clear information to the building facility management. In this scenario, this paper presents the analysis of the data coming from the monitoring system of Palazzo Regione Lombardia [1], which is the high-rise building 
(height of $161 \mathrm{~m}$ ) seat of the regional government. The monitoring system includes accelerometers, clinometers and transducers for environmental variables (e.g. wind strength and direction, temperature).

According to literature, structural monitoring and consequently damage identification could be performed at five different levels of detail: simply detecting the presence of damage and its localization, evaluating the type of damage, quantifying its entity and finally estimating the residual life [2][3].

One of the approaches used for monitoring the present building is based on a continuous modal parameter extraction using the accelerometer signals, coupled to the analysis of the data reckoned by means of clinometers. The modal data are able to describe the dynamics of the structure, while the clinometers are employed to describe its static behaviour. In this paper the possibility of using modal parameters as a damage warning feature is investigated[4].

The modal parameter extraction is carried out by using Operational Modal Analysis (OMA), because the environmental excitation (e.g. wind and traffic), which cannot be measured, is used as source to excite the system[5-7].

At first, the monitoring system installed is briefly described, then, the modal extraction algorithms and their performances are investigated. Two different approaches were tested: the polyreference-least squares complex-frequency domain (pLSCF) method and a method based on the complex mode indicator function (CMIF), which in turn is based on the singular value decomposition (SVD). The two methods are compared in terms of accuracy and bias effects on the estimated modal parameters using the Monte Carlo method. The analysis allowed to choose the best algorithm, as well as optimizing the parameters used in the analysis of the real data. Finally, the first data coming from the modal parameter extraction are presented.

\section{PALAZZO LOMBARDIA AND THE MONITORING SYSTEM}

The "Palazzo Lombardia" building is the first in a series of high rise buildings which have been built in Milano in the last years. It is the current seat for the Regional Government and offices and therefore considered of strategic relevance. The complex is made up of five lower buildings (about 40m high, called Cores 2, 3, 4, 5 and 6), surrounding the high-rise Tower (Core 1), which scored, at the time of construction, the new height record in Italy. The monitoring system is targeted to control core 1 tower and is capable of handling both dynamic vibration signals and static variables, as well as of the wind conditions. A full description of the system installation and performances can be found in [1]. A schematic representation of the system layout is given in Figure 1.
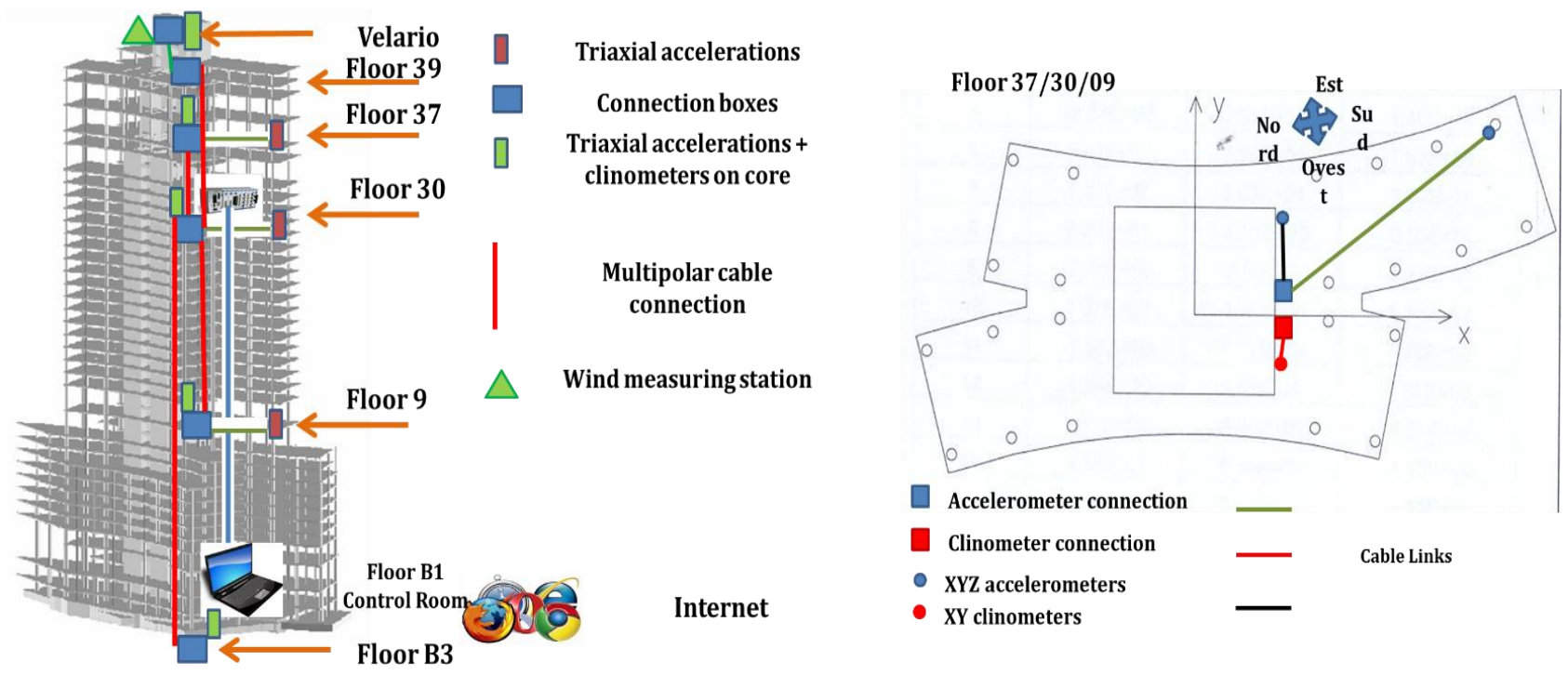

Figure 1 CORE 1 Tower and a scheme of the installed monitoring system 
Five floors are instrumented with clinometers and accelerometers according to the general layout given in Figure 1. The setup was designed in accordance with the dynamic testing results $[6,8]$ in order to be able to identify at least the first three vibration modes and to asses wind comfort issues.

Data acquisition is performed 24h per day with a final sampling frequency of $250 \mathrm{~Hz}$ on all channels enough to asses wind comfort, vibration disturbances and provide data to modal analysis purposes [9,10]. A total of 24 high sensitivity piezo accelerometers and 10 clinometers are installed together with a wind measurement station on top of the building. A new data file is generated every 10 minutes ready to be analysed.

In order to be able to handle such an amount of data a continuous and automated modal extraction algorithm had to be implemented starting from the choice of the identification method and parameters.

\section{MODAL IDENTIFICATION ALGORITHM}

The two algorithms which were taken into account are the pLSCF (Polyreference least square frequency domain) [11] and the FDD [12] (Frequency decomposition), as already mentioned. They are briefly discussed here to provide the most relevant information. More details can be found in the referenced works.

With regard to pLSCF, one of its main properties is that it provides clean and easy-to-interpret stabilization diagrams and this reduces the amount of complexity for its use as well as difficulties for getting reliable results. This has caused the pLSCF to become the industrial standard for modal analysis at the present time. pLSCF is a least-squares approach in frequency and can be used in OMA. In this case the inputs to the method are the positive power-spectra of the system responses.

The second algorithm taken into consideration is the FDD. As mentioned, it is based on SVD. SVD is a linear algebra technique that can achieve factorization of a complex matrix. FDD identification method works by decomposing the power spectral density (PSD) matrix in its principal components, at each spectral line. More details can be found in the wide referenced literature (e.g.).

There are two parameters which must be considered as inputs when using both mentioned OMA identification approaches: the frequency resolution $\mathrm{R}$ of the power-spectra and the number of averages $\mathrm{N}$ used to calculate them. It is known in the literature that a narrow frequency resolution allows to improve the accuracy of the modal identification. It is also easy to understand that the higher the number of averages is, the cleaner the power-spectra will be. This would suggest to increase the time-length of the acceleration signals used to calculate the power-spectra. Indeed, if $T_{t}$ is the total time of the acceleration time-histories used for the modal extraction, and if the whole time-records are divided into $\mathrm{N}$ sub-records of time-length $t_{t}$ (with no overlap), it results that:

$$
R=\frac{1}{t_{\mathrm{t}}}=\frac{N}{T_{\mathrm{t}}} \Rightarrow T_{\mathrm{t}}=\frac{N}{R}
$$

Therefore, $\mathrm{T}_{\mathrm{t}}$ must be increased in order to both increase $\mathrm{N}$ and decrease $\mathrm{R}$.

However, $T_{t}$ cannot be increased indefinitely because the structure changes its behaviour in time: e.g. the modal parameters of a structure change between day and night due to thermal shifts. According to these points, a maximum possible value for $\mathrm{T}_{\mathrm{t}}$ was fixed equal to $10800 \mathrm{~s}$ (i.e. 3 hours), which is a time span over which an initial data check did not evidence any significant effect of the environmental conditions. Once this threshold was fixed, the effects of different values of $\mathrm{R}$ and $\mathrm{N}$ were studied by means of MCMC simulations. 


\section{MARKOV CHAIN MONTE CARLO SIMULATIONS}

The comparison of pLSCF and FDD was carried out by means of the MCMC method. Time-histories of accelerations were generated numerically with the goal to make them as close as possible to those collected by the accelerometers placed in the building. Then these numerical time-records were provided as inputs to the two algorithms.

These simulated signals were generated by means of a modal model of the structure, considering the first three modes Table 1. The PSDs of the generated signals were made as close as possible to the PDSs of the actual signals collected by the accelerometers. The reference actual PSDs were chosen from a day with very low wind in order to test the case with the poorest signal-to-noise ratio.

\begin{tabular}{cccccc}
\hline $\begin{array}{c}\text { First } \\
\text { eigenfrequency }\end{array}$ & First mode shape & $\begin{array}{c}\text { Second } \\
\text { eigenfrequency }\end{array}$ & $\begin{array}{c}\text { Second mode } \\
\text { shape }\end{array}$ & $\begin{array}{c}\text { Third } \\
\text { eigenfrequency }\end{array}$ & Third mode \\
& & & & shape \\
\hline $0.32 \mathrm{~Hz}$ & Bending (east- & $0.40 \mathrm{~Hz}$ & Bending (north- & $0.63 \mathrm{~Hz}$ & Torsion \\
& west direction) & & south direction) & & \\
& & & & &
\end{tabular}

Table 1: Reference parameters for the Monte Carlo simulations

The effect of the electrical noise due to the transducers, cables, etc. was taken into account as well. Figure 2 shows a comparison between a PSD of a simulated signal and a real one. The agreement is satisfactory. This figure also shows that the value of the numerical PSD curve far from the resonances is close to the experimental one. This proves that the amount of random noise added to the numerical signals was correct.

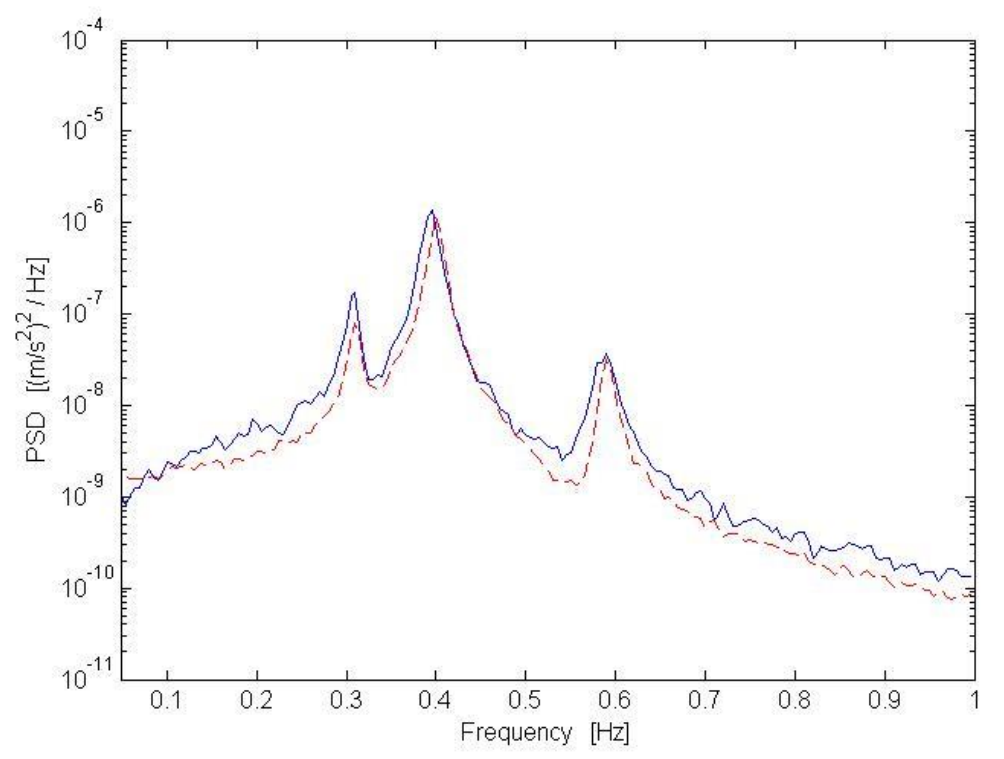

Figure 2: Experimental (solid curve) and numerical (dashed curve) PSD for an accelerometer on floor 30 in case of low wind.

The signal generation for each configuration tested (i.e. fixed values of $\mathrm{N}$ and $\mathrm{R}$ ) was repeated 300 times. Then, modal identification was carried out for each iteration. The focus of the analysis was on the estimation of eigenfrequencies and mode shape components. The results related to mode shapes were described by a synthetic index: the modal assurance criterion (MAC) between the identified mode shapes and the reference ones used in the modal model.

This procedure allowed us to build statistical populations for the following indexes: 
- $\quad$ errors between estimated and reference eigenfrequency values. These errors accounts for bias on the estimations by computing the population mean values $\mu$ and for the dispersion of the estimation by employing the standard deviation $\sigma$;

- $\quad$ MAC values. The mean MAC value $\mu$ accounts for bias, while again dispersion is related to the standard deviation $\sigma$ of the MAC populations.

The trends of these mean and standard deviation values were investigated as function of two input variables: the number of averages $\mathrm{N}$ and the frequency resolution $\mathrm{R}$ employed to calculate the power-spectra provided as inputs to the modal identification algorithms. Table 2 shows the limit values of $\mathrm{R}$ and $\mathrm{N}$ tested in the MCMC simulations.

\begin{tabular}{lcc}
\hline Tested values of $T_{\mathrm{t}}[\mathrm{s}]$ & Tested values of $N$ & Tested values of $\mathrm{R}[\mathrm{mHz}]$ \\
\hline From 1000 to 10800 & From 13 to 72 & From 3.3 to 13 \\
\hline Table 2: Values of $\boldsymbol{T}_{\mathrm{t}}, \mathbf{R}$ and $\mathbf{N}$ tested in the Monte Carlo simulations.
\end{tabular}

Table 2: Values of $T_{t}, R$ and $\mathbf{N}$ tested in the Monte Carlo simulations.

Figure 3 to Figure 6 show the results in terms of $\mu$ and $\sigma$ for the first three modes of the building as function of the frequency resolution and the number of averages. Particularly, Figure 3 and Figure 4 show the results for pLSCF, while Figure 5 and Figure 6 for FDD.
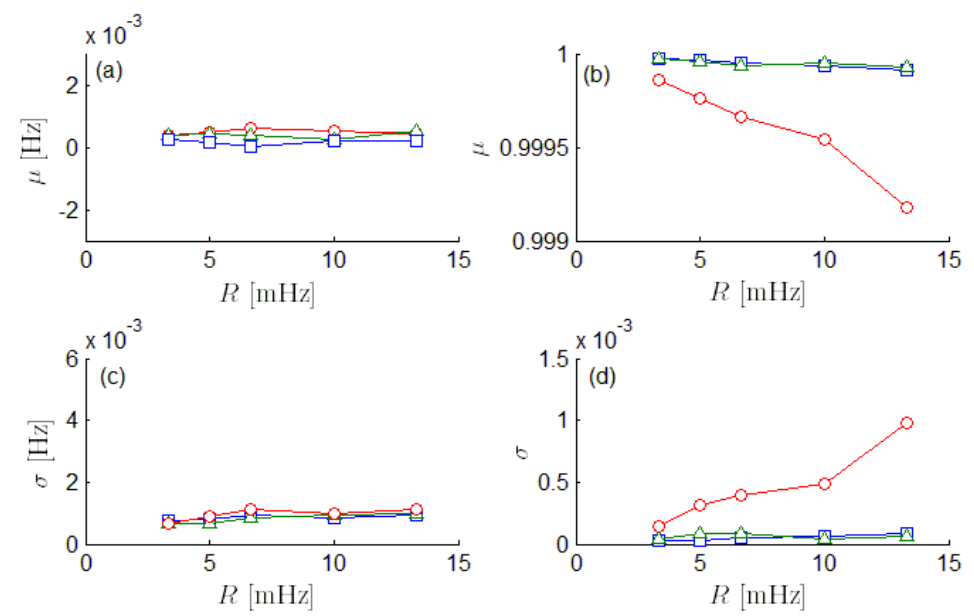

Figure 3: Results of the MCMC simulations for pLSCF as function of $R(N=36)$ : mean value of the error for the eigenfrequency estimation (a), mean value of the MAC (b), $\sigma$ of the error for the eigenfrequency estimation (c), and $\sigma$ for the MAC (d). Curves with squares $(\square)$ for the first mode, with circles $(0)$ for the second mode, with triangles $(\Delta)$ for the third mode. 

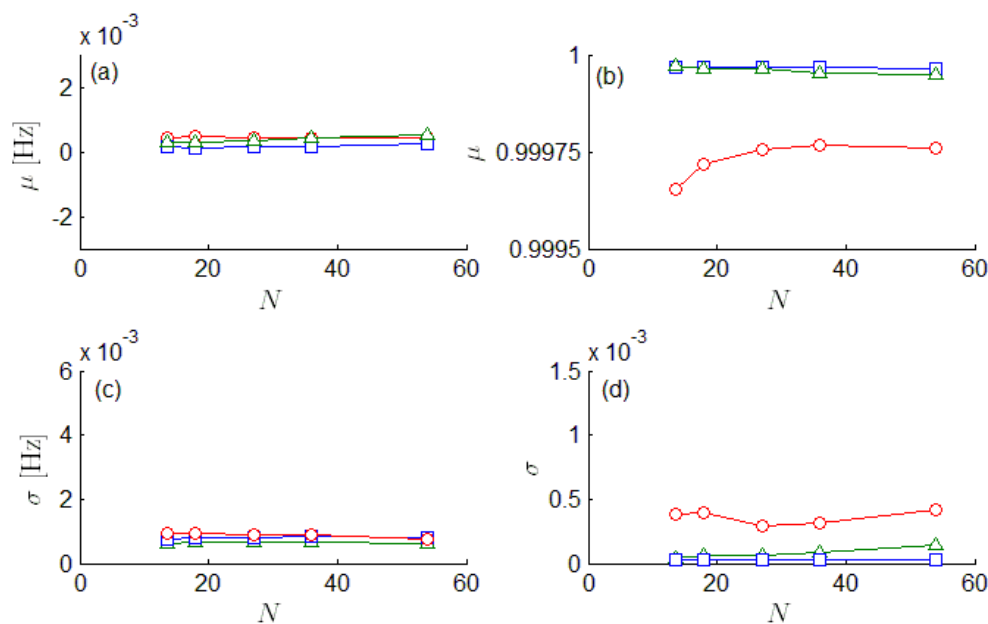

Figure 4: Results of the MCMC simulations for pLSCF as function of $N(R=5 \mathrm{mHz})$ : mean value of the error for the eigenfrequency estimation (a), mean value of the MAC (b), $\sigma$ of the error for the eigenfrequency estimation (c), and $\sigma$ for the MAC (d). Curves with squares $(\square)$ for the first mode, with circles $(0)$ for the second mode, with triangles $(\Delta)$ for the third mode.
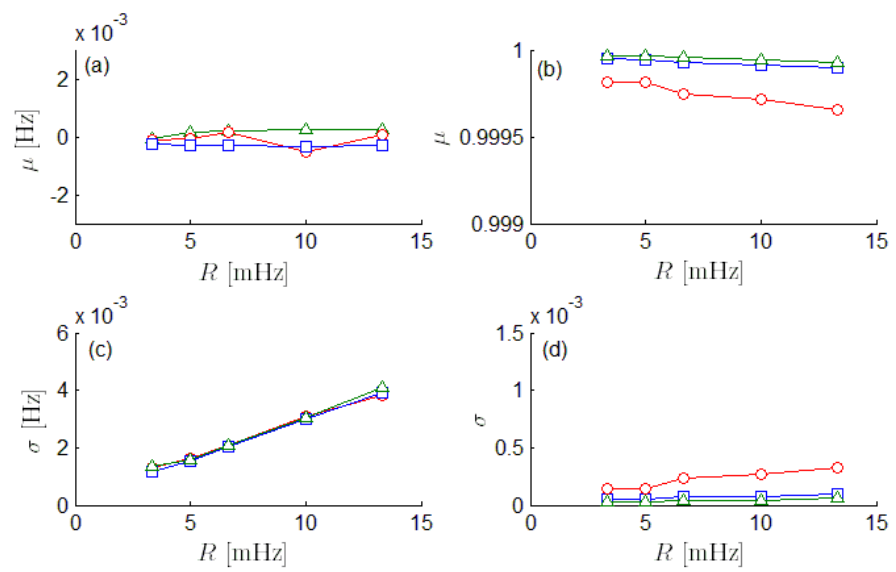

Figure 5: Results of the MCMC simulations for FDD as function of $\mathrm{R}$ : mean value of the error for the eigenfrequency estimation ( $N=48$ ) (a), mean value of the $\operatorname{MAC}(N=36)(b), \sigma$ of the error for the eigenfrequency estimation ( $N=48)(c)$, and $\sigma$ for the MAC ( $N=36)$ (d). Curves with squares $(\square)$ for the first mode, with circles $(0)$ for the second mode, with triangles $(\Delta)$ for the third mode. 

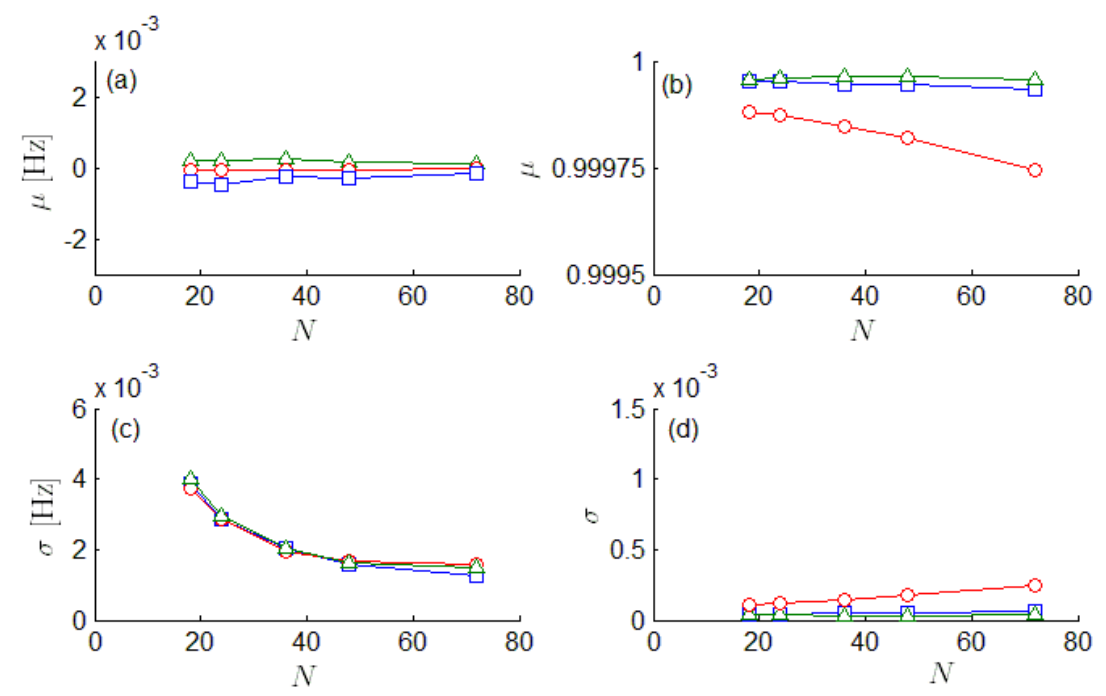

Figure 6: Results of the MCMC simulations for FDD as function of $N(R=5 \mathrm{mHz})$ : mean value of the error for the eigenfrequency estimation (a), mean value of the MAC (b), $\sigma$ of the error for the eigenfrequency estimation (c), and $\sigma$ for the MAC (d). Curves with squares $(\square)$ for the first mode, with circles $(0)$ for the second mode, with triangles $(\Delta)$ for the third mode.

The analysis of these figures suggests the use of pLSCF for estimating the eigenfrequencies. Conversely, the FDD is more accurate for identifying mode shapes. Choosing a mixed approach allows to improve the accuracy associated to the estimated modal quantities. The MCMC simulation also allowed to find reliable data about the effect of frequency resolution and number of averages of the power-spectra and thus to choose properly their values (see Table 3).

$\begin{array}{lll}\text { Chosen value of } T_{\mathrm{t}}[\mathrm{s}] & \text { Chosen value of } N & \text { Chosen value of } R[\mathrm{mHz}]\end{array}$

$\begin{array}{lll}7200 & 36 & 5\end{array}$

Table 3: Chosen values of $T_{t}, R$ and $N$ to be used in OMA with real signals.

The bias values associated to the identified eigenfrequencies are very low, as evidenced by Figure 3a and Figure 4a. Instead, the expected values of the dispersion associated to the identified eigenfrequencies are gathered in Table 4 and can be used as an estimation of the uncertainty of the identification method [13].

\begin{tabular}{ccc}
$\begin{array}{c}\sigma \text { on the first } \\
\text { eigenfrequency }\end{array}$ & $\begin{array}{c}\sigma \text { on the second } \\
\text { eigenfrequency }\end{array}$ & $\begin{array}{c}\sigma \text { on the third } \\
\text { eigenfrequency }\end{array}$ \\
\hline $0.8 \mathrm{mHz}$ & $0.8 \mathrm{mHz}$ & $0.6 \mathrm{mHz}$
\end{tabular}

Table 4: Expected values of $\sigma$ on the eigenfrequency estimations.

It is remarked that, for the configuration of values of Table 3, the MCMC test was repeated with a higher number of simulations (using an adaptive version of the MCMC) to assess statistical reliability. The results obtained are almost the same of those already shown with 300 simulations.

FIRST RESULTS FROM THE REAL DATA

The values of $\mathrm{R}$ and $\mathrm{N}$ chosen with the MCMC simulations, as well as the use of the mixed approach pLSCF/FDD, allowed to develop an automatic OMA identification system. To do this, an automated data-check procedure was also developed. 
Relying on the use of the Skewness coefficient and peak-peak values of the signals, corrupted time records (e.g. due to saturation, lightning, transducer damage) are automatically discarded before modal identification.

The automated extraction was applied to eight months of vibration data coming from the installed monitoring system. At first the uncertainty interval equal to $\pm 2 \sigma$ (see Table 4 ) around the identified eigenfrequency value for the first mode is shown in Figure 7. This $\pm 2 \sigma$ interval expresses a level of confidence of about $95 \%$. The width of the uncertainty intervals is clearly overestimated. This is probably due the fact that the value of $\sigma$ was estimated through MCMC considering a day with very low wind and consequently a very bad signal to noise ratio.

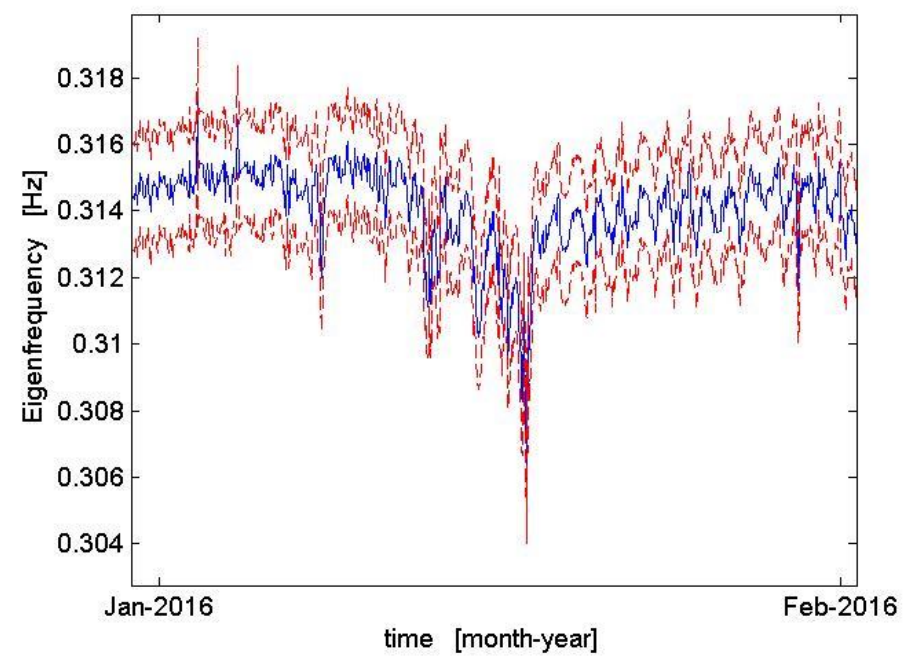

Figure 7: Trend of the first eigenfrequency (solid blue curve) and $\pm 2 \sigma$ interval (dashed red curves).

Figure 8 to Figure 10 show the trend of the first three eigenfrequencies of the structure. Instead, Figure 11 shows the MAC trend for the first mode. The changes of the eigenfrequencies are always lower than $5 \%$.

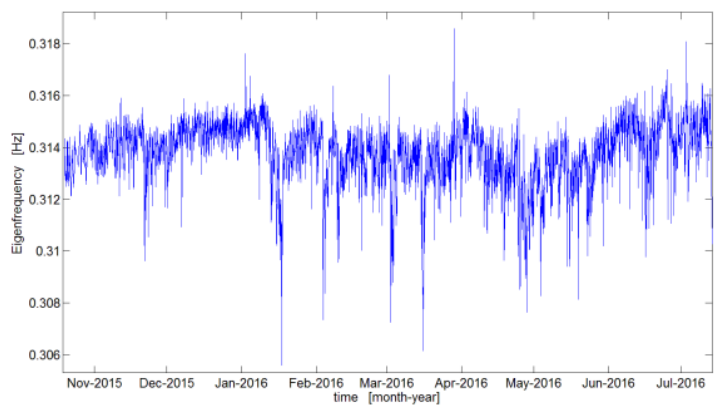

Figure 8: Time trend of the first eigenfrequency.

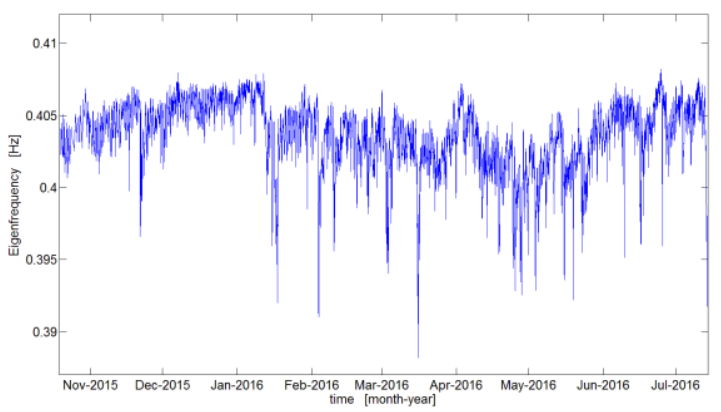

Figure 9: Time trend of the second eigenfrequency.

The wind speed is proved to be able to change the eigenfrequency values significantly. Indeed, the spikes towards zero in Figure 8 to Figure 10 are always related to the presence of an increased value of the root mean square (RMS) of the acceleration signals (the RMS is calculated on the frequency band of the considered mode). This RMS is strongly correlated with the wind speed. 


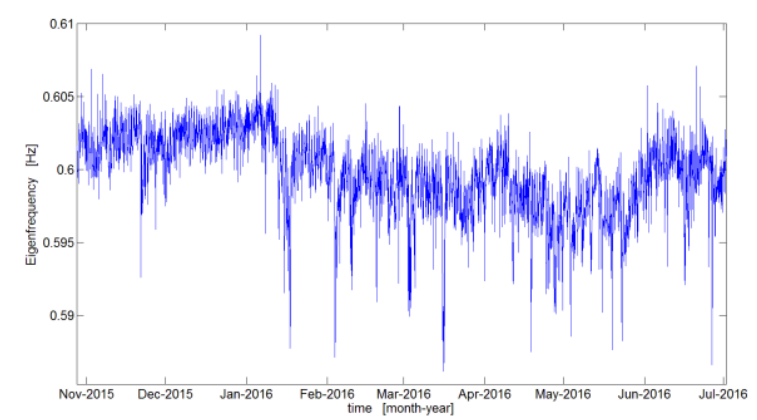

Figure 10: Time trend of the third eigenfrequency.

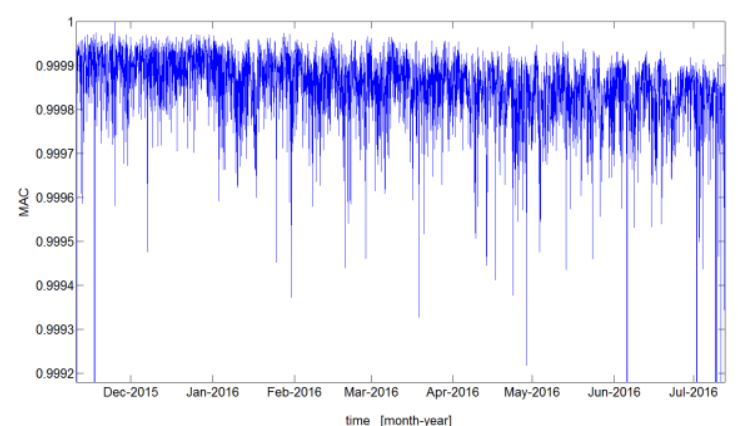

Figure 11: Time trend of the MAC of the first mode.

A slight descending trend is put into evidenze for the MAC coefficient even if looking at the scale in Figure 11 it can be noticed that this variability is in the order of $1 \mathrm{E}-4$, which is less than the identification ucertainty.

\section{CONCLUSION}

The paper has described the installed monitoring system and a proposed data analysis strategy for Palazzo Lombardia, one of the tallest building in Milano. The layout of the system has been presented, and the choices related to the used sensors and their locations are discussed.

An approach based on automatic and continuous modal parameters extraction has then been presented for structural health monitoring purposes. The main features of the automatic modal analysis software used for modal identification are then provided, explaining how Monte Carlo simulations were used to optimise it.

This automatic identification system allowed to produce the first plots of the trends of the modal parameters of the building along eight months of monitoring. These modal parameter trends will allow to develop a statistical model to describe the relationship between the first three eigenfrequencies of the building and a number of input variables representative of the environmental conditions. The statistical model will be used to detect anomalous trends indicating a change in the structure.

\section{REFERENCES}

[1] Berardengo M, Cigada A, Manzoni S and Vanali M 2016 Design and Installation of a Permanent Monitoring System for Palazzo Lombardia in Milano, Italy VII Eur. Congr. Comput. Methods Appl. Sci. Eng. - ECCOMAS Congr. 2016 - Crete Isl. (Greece), 5-10 June $201613640-51$

[2] Farrar C R and Worden K 2012 Structural Health Monitoring - A Machine Learning Perspective (Wiley)

[3] Mesquita E, Antunes P, Coelho F, André P, Arede A and Varum H 2016 Global overview on advances in structural health monitoring platforms J. Civ. Struct. Heal. Monit. 6 461-75

[4] Yang Z and Wang L 2010 Structural damage detection by changes in natural frequencies J. Intell. Mater. Syst. Struct. 21 309-19

[5] Rainieri C and Fabbrocino G 2014 Operational modal analysis of civil engineering structures (New York: Springer)

[6] Busca G, Cigada A, Mola E, Mola F and Vanali M 2014 Dynamic testing of a helicopter landing pad: comparison between operational and experimental approach J. Civ. Struct. Heal. Monit. 4 133-47

[7] Ewins D J 2000 Modal testing: theory, practice and application (Baldock: Research studies press Ltd.)

[8] Cigada A, Mola E, Mola F, Stella G and Vanali M 2014 Dynamic Behavior of the Palazzo Lombardia Tower : Comparison of Numerical Models and Experimental Results J. Perform. Constr. Facil. 28(3) 491-501

[9] Mendis P, Ngo T, Haritos N, Hira A, Samali B and Cheung J 2007 Wind loading on tall buildings EJSE Spec. Issue 


\section{Load. Struct. 3 41-54}

[10] Griffis L G 1993 Serviceability limit states under wind load Eng. J. 30 1-16

[11] Peeters B, Auweraer H Van Der, Guillaume P and Leuridan J 2004 The PolyMAX frequency-domain method : a new standard for modal parameter estimation? Shock Vib. 11 395-409

[12] Wang T, Celik O, Catbas F N and Zhang L M 2016 A frequency and spatial domain decomposition method for operational strain modal analysis and its application Eng. Struct. 114 104-12

[13] JCGM 100:2008 2008 Evaluation of measurement data — Guide to the expression of uncertainty in measurement 